\title{
Association between Dietary Inflammatory Index and Type 2 diabetes mellitus in Xinjiang Uyghur autonomous region, China
}

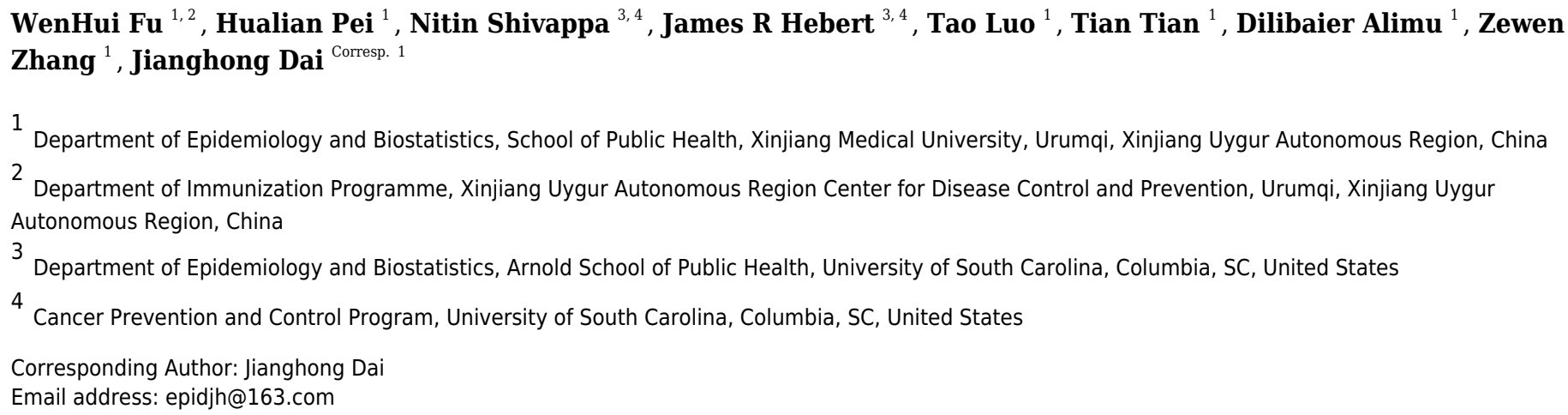

Background Diet and inflammation have both been studied in relation to type 2 diabetes mellitus (T2DM). The aim of this cross-sectional study was to examine the association between the dietary inflammatory index $\left(\mathrm{DII}^{\circledR}\right)$ and T2DM. Methods Subjects were adults enrolled in the baseline study of the Xinjiang multi-ethnic natural population cohort and health follow-up study from January to May 2019. The study involved 5105 subjects $(58.7 \%$ men) between 35 and 74 years of age. The DII score was calculated from a data obtained via a food frequency questionnaire consisting of 127 food items. Results Logistic regression analyses were used to estimate the odds ratios (ORs) and $95 \%$ confidence intervals $(95 \% \mathrm{Cls})$ of DII in relation to T2DM. After adjusting for potential confounders, compared to subjects in the $1^{\text {st }}$ DII quintile, subjects in the $5^{\text {th }}$ quintile (i.e., with the most pro-inflammatory diet) had higher odds of T2DM ( $\mathrm{OR}=3.27,95 \% \mathrm{Cl}: 2.38,4.50 ; p<0.001)$. Conclusions Our results suggest that a pro-inflammatory diet is associated with a higher risk of T2DM in this population of Chinese adults. 


\section{Association between Dietary Inflammatory Index and Type 2 diabetes mellitus in Xinjiang Uyghur autonomous region, China}

Wenhui $\mathrm{Fu}^{1,2}$, Hualian Pei ${ }^{1}$, Nitin Shivappa ${ }^{3,4}$, James R. Hebert ${ }^{3,4}$, Tao Luo ${ }^{1}$, Tian Tian ${ }^{1}$, Dilibaier Alimu ${ }^{1}$, Zewen Zhang ${ }^{1}$ and Jianghong Dai ${ }^{1}$

${ }^{1}$ Department of Epidemiology and Biostatistics, School of Public Health, Xinjiang Medical University, Urumqi, Xinjiang, China

2 Department of Immunization Programme, Xinjiang Uygur Autonomous Region Center for Disease Control and Prevention, Urumqi, Xinjiang, China

${ }^{3}$ Cancer Prevention and Control Program, University of South Carolina, Columbia, SC, USA

${ }^{4}$ Department of Epidemiology and Biostatistics, Arnold School of Public Health, University of South Carolina, Columbia, SC, USA

Corresponding Author:

Jianghong Dai ${ }^{1}$

No.393 Xinyi Road, Xinshi District, Urumqi, the Xinjiang Uygur Autonomous Region, 830011, China

Email address: epidjh@163.com 


\section{Abstract}

2 Background Diet and inflammation have both been studied in relation to type 2 diabetes

3 mellitus (T2DM). The aim of this cross-sectional study was to examine the association between

4 the dietary inflammatory index (DII $\left.{ }^{\circledR}\right)$ and T2DM.

5 Methods Subjects were adults enrolled in the baseline study of the Xinjiang multi-ethnic natural

6 population cohort and health follow-up study from January to May 2019. The study involved

75105 subjects ( $58.7 \%$ men) between 35 and 74 years of age. The DII score was calculated from a

8 data obtained via a food frequency questionnaire consisting of 127 food items.

9 Results Logistic regression analyses were used to estimate the odds ratios (ORs) and 95\%

10 confidence intervals (95\% CIs) of DII in relation to T2DM. After adjusting for potential confounders, compared to subjects in the $1^{\text {st }}$ DII quintile, subjects in the $5^{\text {th }}$ quintile (i.e., with the most pro-inflammatory diet) had higher odds of T2DM ( $\mathrm{OR}=3.27,95 \% \mathrm{CI}: 2.38,4.50 ; p<0.001)$.

13 Conclusions Our results suggest that a pro-inflammatory diet is associated with a higher risk of T2DM in this population of Chinese adults.

Keywords: Dietary Inflammatory Index; Type 2 diabetes mellitus; China

\section{Introduction}

Type 2 diabetes mellitus (T2DM) is a chronic metabolic disease (Zuo et al. 2014). Current projections estimate that between 2017 and 2045 the number of people suffering from diabetes will increase more than 50\%, with the vast majority diagnosed with T2DM (Cho et al. 2018). In 2017, the prevalence of diabetes in China was 11.2\% (Wang H P et al. 2020).

Of the many metabolic and lifestyle factors that are known to affect the development and complications of T2DM, inflammation has been gaining attention for its role in T2DM pathogenesis (Dregan et al. 2014). Overexpression of proinflammatory cytokines, such as Interleukin-1 $\beta$ (IL-1 $\beta$ ), Interleukin-6 (IL-6), and Tumor necrosis factor- $\alpha$ (TNF- $\alpha$ ), induce chronic inflammation in T2DM(JOHN C. PICKUP 2004; Mahlangu et al. 2019; Pradhan A D et al. 2001), although the specific mechanism remains unclear. There are both modifiable and unmodifiable factors associated with inflammation; dietary factors are potentially modifiable and, therefore, have received a great deal of attention (Moazzen et al. 2020).

The effect of diet on inflammation can be assessed based on food-group, nutrition, or analysis of dietary patterns (Barbaresko et al. 2013). Because humans consume many nutrients through a 
32 variety of foods, a person's inflammatory status can be influenced by the balance between

33 nutrients and other dietary components that promote or inhibit inflammation (Minihane et al.

34 2015). Therefore, in order to illustrate the complex effects of dietary components on

35 inflammatory status, a better assessment method is needed to evaluate the impact of the overall

36 diet on individual inflammatory status.

$37 \quad$ The dietary inflammatory index $\left(\mathrm{DII}^{\circledR}\right)$ has been developed to characterize the inflammatory

38 potential of the diet (Cavicchia et al. 2009). The design of this index included careful review and

39 scoring of results from peer-reviewed research studies from human populations, qualifying cell

40 culture experiments, and animal experiments (Shivappa et al. 2014). The validity of the DII has

41 been demonstrated using different inflammatory biomarkers (Kotemori et al. 2020; Tabung et al.

42 2015). In addition, it is associated with components of metabolic syndrome (Ferreira et al. 2019)

43 and a wide variety of chronic diseases(Phillips et al. 2019).

44 Xinjiang is a multi-ethnic region where the Han, Uygur, and Kazak groups account for more

45 than $90 \%$ of the total population. Because of differences in customs and living environments,

46 there is a certain heterogeneity in dietary structure among these different ethnic groups.

47 According to the physical examination data for all citizens in Xinjiang in 2018, the detection rate

48 of T2DM varied among different populations. Thus, the aim of this study was to explore the

49 relationship between the DII and T2DM in different participants in the Xinjiang multi-ethnic 50 natural population.

\section{Materials \& Methods}

53 Study population

54 The Xinjiang multi-ethnic natural population cohort construction and health follow-up study is a 55 population-based prospective study designed by our group and implemented from January to 56 May 2019. Participants in this study were members of a subsample in the baseline study, aged 57 35-74 years, and living in Yili Prefecture, Xinjiang Province in China.

58 Potential participants were invited if they met the following criteria for inclusion: (1) 59 participants of the original cohort; (2) provided basic information, including diet and physical 60 activity; (3) provided stored serum samples and blood analysis data. A total of 8367 adults, aged 61 35-74 years were enrolled. For the present analysis, we excluded participants with no fasting 62 glucose data $(\mathrm{n}=2166)$, then we excluded participants with missing information on important 
63 covariates $(n=797)$ or with incomplete anthropometric data $(n=299)$. Finally, a total of 5105

64 subjects were included in our study (Figure 1).

65 All recruited subjects gave written informed consent for inclusion before they participated in

66 the study. The study was conducted in accordance with the Declaration of Helsinki, and the

67 protocol was approved by the Ethics Committee of Ethics Committee of Xinjiang Uygur

68 Autonomous Region Academy of Traditional Chinese Medicine(2018XE0108).

69

70 Dietary Assessment

71 Habitual diet over the past year was assessed using the interviewer-administered food frequency

72 questionnaire (FFQ)(Hailili et al. 2020). Referring to the dietary questionnaire of Xinjiang multi-ethnic

73 natural population cohort construction and health follow-up study and combining the characteristics of a

74 typical Xinjiang diet, the final questionnaire included 127 foods items. Consumption frequency

75 of each food was queried on a daily, weekly, or monthly basis and converted into daily intake.

76 The FFQs were collected by trained personnel.

77

78 DII Score

79 Dietary data from the FFQs were used to calculate DII scores for each participant. The design 80 and development of the DII has been described previously. Briefly, DII scores were calculated

using a scoring algorithm, which was based on a review of 1,943 articles between 1950 and 2010

82 that reported on 45 different food parameters that were found to be associated with six

83 inflammatory biomarkers (IL-1 $\beta$, IL-4, IL-6, IL-10, and C-reactive protein). Each study was

84 assigned a "food parameter-specific inflammatory effect score." If the food parameter was pro-

85 inflammatory, it was assigned $\mathrm{a}+1$. If it was anti-inflammatory, it was assigned $\mathrm{a}-1$. If the

86 parameter did not change significant in terms of inflammation biomarkers, they were assigned a

87 zero. The specific calculation steps were as follows: (1) dietary intake data were collected from

88 study subjects using the FFQ; (2) dietary intake data were transformed to the food ingredients

89 contained in the DII scale, according to the latest China Food Composition Table; (3) dietary

90 intake data were compared with the global standard dietary intake database and a Z-score was

91 calculated for each nutrient in the study according to the mean and standard deviation of each

92 nutrient intake; (4) Z-scores were converted to centered proportions; 5) food parameter specific

93 centered proportion is multiplied by the specific inflammatory effect score of each nutrient to 
94 derive food parameter specific DII score; (5) DII scores were then summed across over all food 95 parameters to obtain an individual's specific DII score.

96 In our study, out of 45 possible foods, individual DII scores were calculated using 27 foods 97 and nutrients for which we obtained intake data from FFQ. The 27 foods and nutrients included 98 carbohydrate, protein, fat, alcohol, fiber, cholesterol, saturated fatty acid, mono-unsaturated fatty 99 acid, poly-unsaturated fatty acid, omega 3 fat, omega 6 fat, trans fat, niacin, thiamin, riboflavin, 100 vitamin B12, vitamin B6, iron, magnesium, zinc, vitamin A, vitamin C, vitamin E, folic acid, 101 beta carotene, garlic, and onion.

102

103

Biomarker evaluation

104 Fasting venous blood samples were collected from the anterior cubital vein of each participant 105 (fasting time $\geqslant 8 \mathrm{~h}$ ). Small portions of serum were stored in refrigerated bottles and transported to 106 laboratories, where they were then stored in equal portions at $-80^{\circ} \mathrm{C}$ for further analysis. 107

108

109

110

111

112

113

114

115

116

117

118

119

120

121

122

123

\section{Definition of T2DM}

For the present study, participants who had previously been diagnosed with diabetes at the county hospital level or above were labeled as "previously diagnosed." Subjects with fasting glucose concentration $\geqslant 5.55 \mathrm{mmol} / \mathrm{L}$ during the study period were defined as having fasting glucose and/or HbA1c values that met the diagnostic criteria for T2DM and were also defined as T2DM patients.

\section{Anthropometric Assessment}

Weight and height were measured while wearing only light clothing, no shoes, and using standard scales. Body mass index (BMI) was calculated as weight $/$ height ${ }^{2}\left(\mathrm{~kg} / \mathrm{m}^{2}\right)$ and classified according to the criteria of the Blue Book on Obesity Prevention and Control in China: underweight $\left(<18.5 \mathrm{~kg} / \mathrm{m}^{2}\right)$, normal weight $\left(18.5-23.9 \mathrm{~kg} / \mathrm{m}^{2}\right)$, overweight $\left(24-27.9 \mathrm{~kg} / \mathrm{m}^{2}\right)$, or obese $\left(\geqslant 28 \mathrm{~kg} / \mathrm{m}^{2}\right)$. 


\section{Other Participant Characteristics}

125 Investigators used a standardized questionnaire to collect information regarding the participants'

126 demographic characteristics(Hailili et al. 2020)(e.g. age, sex, education, marital status), self-

127 perception of body weight, past medical history, current medication use, lifestyle information

128 (e.g., physical activity, smoking status, alcohol consumption, etc.) and information on

129 reproductive history (for females).

130

\section{Statistical Analysis}

132 We performed a descriptive analysis of the main characteristics of interest to assess compliance 133 with the model's assumptions. We analyzed the characteristics difference between diabetic and 134 non-diabetic subjects. $P$-values were determined using a student's t-test for continuous variables 135 and Chi-square test for categorical variables. Moreover, Kolmogorov-Smirnov test was used to check the normality. Categorical variables were presented as frequencies of occurrence and percentages, and continuous variables were presented as the mean and standard deviation (SD). One-way analysis of variance (ANOVA) was used to examine whether there were differences across DII quintiles, while the chi-square test was used to assess the distribution of qualitative variables over DII quintiles.

To evaluate the magnitude of the association between the DII and diabetes, we used a simple univariable (unadjusted) and multivariable logistic regression analysis adjusted for baseline characteristics (sex, age, ethnicity, physical activity, smoking, education, alcohol intake, body mass index). In order to asses possible effect modification, analyses stratified by physical activity, BMI, ethnic and age were performed. $P$-Interaction was calculated using the multiplicative interaction term. We tested the significance using a likelihood ratio test by comparing a model with the main effects of each intake and the stratified variable and the reduced model interaction terms with only the main effect. All $p$-values presented are two-tailed; $p<0.05$ was considered significant.

\section{Sensitivity analysis}

Several sensitivity analyses were performed. First, we adjusted different models when multiple 
154 unmeasured confounding. E-values were calculated by the online E-value calculator

155 (https://mmathur.shinyapps.io/evalue/).

156 All statistical analyses were performed using SPSS 21.0 software and SAS ${ }^{\circledR} 9.4$ (SAS Institute

157 Inc., Cary, NC). All $p$ values were based on two-sided tests.

158

\section{Results}

160 The baseline characteristics of participants are shown in Table 1. A total of 5105 subjects $(58.7 \%$ 161 male) were included in the present analysis. The main age of the participants ranged from 45 to 16260 years, the mean DII of all participants was 0.81 , and the overall prevalence of T2DM was $1638.2 \%$. Furthermore, the mean DII was significant higher in participants with $\operatorname{T} 2 \mathrm{DM}(p<0.001)$. 164 According to DII score quintiles, the participants with the most pro-inflammatory diets were 165 166 167 168

169 significant older $(p=0.004)$ and more likely to be obese $(p=0.03)$. These participants also had lower educational status, lower physical activity level, and higher blood glucose. The distribution of DII by ethnicity showed a higher proportion of ethnic minorities with pro-inflammatory diets (Table 2).

Whether using DII as a continuous or categorical variable, the correlation between T2DM and DII was evident. After adjusting for ethnicity and sex, the odds of having T2DM across all DII score quintiles were 1.00, 1.12, 1.17, 1.18, and 3.37 (95\% CI: 2.46, 4.63; $p<0.001)$. Finally, after additional adjustment for variables, we observed that subjects with the most pro-inflammatory diets had higher odds of having T2DM (OR: 3.27, 95\% CI: 2.38, 4.50; $p<0.001$ ), compared to individuals in the lowest DII quintile (Table 3, Figure 2). The results of the stratified and interaction analysis are shown in Figure 3 and supplemental Table 4. The correlation between DII and T2DM was stronger among subjects with low physical activity level ( $\left.\mathrm{OR}_{\mathrm{Q} 5} \mathrm{vs}_{\mathrm{Q} 1}: 5.62,95 \% \mathrm{CI}: 3.00,10.51\right)$, obese subjects $\left(\mathrm{OR}_{\mathrm{Q}_{5}} \mathrm{vs}_{\mathrm{Q} 1}: 4.62,95 \% \mathrm{CI}\right.$ : $2.50,8.56)$, Uyghurs $\left(\mathrm{OR}_{\mathrm{Q} 5}\right.$ vs $\left.\mathrm{Q}_{1}: 4.11,95 \% \mathrm{CI}: 1.85,9.14\right)$ and participants $\geqslant 55$ years $\left(\mathrm{OR}_{\mathrm{Q} 5}\right.$ vs $\left._{\mathrm{Q} 1}: 5.14,95 \% \mathrm{CI}: 2.79,9.47\right)$.

Additionally, we conducted a sensitivity analysis for unmeasured confounding in this study using E-values (Linden et al. 2020). E-values can be used to estimate the minimum correlation strength associated with outcome indicators for unmeasured confounders that can explain away the research results, so as to evaluate the robustness of the association between exposure factors and outcome indicators, and when the E-value is relatively large, it may provide strong evidence 
185 to support the relationship. In our study, the E value of the point estimate was 5.99 that is higher 186 than the observed risk ratio 3.27, so it provided the evidence to support the relationship between 187 DII and T2DM (Supplemental Figure 4).

\section{Discussion}

189 In the baseline survey of a Xinjiang multi-ethnic cohort study, we found an positive correlation

190 between DII and T2DM. Those with the most pro-inflammatory diets had a higher risk of 191 developing T2DM compared to participants with the lowest quintile (most anti-inflammatory 192 diets). This increased risk related to pro-inflammatory diet was independent of other diabetes risk 193 factors. This finding is in accord with prior publications from the Mexico City Diabetes Mellitus 194 Survey (Denova-Gutierrez et al. 2018), where subjects in the highest DII quintile were observed 195 to be about three times more likely to develop T2DM than those in the lowest DII quintile.

In this study, we used the previously derived and validated DII scores to assess the effect of a pro-inflammatory diet on T2DM. The DII score for The Xinjiang population included 27 food parameters, with a mean of 0.81 . The mean of DII in our study was slightly higher than in other studies. For example, the mean value of DII in a large French prospective cohort of women was 0.06 (Laouali et al. 2019) and a study in a Japanese population reported a mean of DII as -2.07 (Kotemori et al. 2020), both of which are lower than what we observed in our population. This may result from ethnic and culinary differences among participants that were analyzed in our study. Our objects were from Yili prefecture in Xinjiang, which belongs to the area inhabited by ethnic minorities. The diet in this area is mainly composed of pasta, beef, mutton, cream, and other of pro-inflammatory diet components. The intake of vegetables, fruits, and other antiinflammatory diet components is relatively low in this area. This is consistent with previous reports that differences in nutrient intake or food consumption are associated with DII (Alipoor et al. 2019; Ashton et al. 2020). Lower C-reactive protein concentration was associated with higher intake of fruits and vegetables (Shivappa et al. 2019), legumes, nuts, and low-fat dairy products (Ferreira et al. 2019; Lohman et al. 2019). Previous studies have also reported associations between intake of certain nutrients, such as total dietary fiber intake, moderate alcohol consumption (Padin et al. 2019), vitamin E and vitamin C intake (Oliveira et al. 2019), and lower levels of inflammatory markers (Hebert et al. 2014).

214 This research has shown that DII is related to an increased risk of T2DM, a noncommunicable chronic disease of great public health importance. Higher dietary inflammatory 
216 potential increases the risk of obesity and its complications, such as T2DM and cardiovascular 217 disease (Zhong et al. 2017). In our study, we observed that subjects in the highest DII quintile 218 were at greater risk for developing T2DM than subjects in the lowest DII quintile. Our analysis 219 was consistent with previous studies that have assessed the relationship between diet and T2DM. 220 One study among middle-aged South African women indicated that DII is associated with T2DM 221 risk through obesity, particularly central (i.e., abdominal) obesity (Mtintsilana et al. 2019).

222 Studies in pre-diabetes have also found that an anti-inflammatory diet prevents the progression 223 of pre-diabetes into frank diabetes (Jacobo-Cejudo et al. 2017). Multiple intervention studies 224 have shown that an anti-inflammatory diet high in carbohydrates, low in fat, and relatively high 225 in fiber reduces the incidence of T2DM by $50 \%$, possibly because a high-fat diet increases 226 inflammatory cytokines, leading to insulin resistance and high blood sugar (McGeoch et al. 227 2013).

228 The link between DII and T2DM can be explained by the effect of a pro-inflammatory diet on 229 insulin resistance, which is associated with the inflammatory state (Roden \& Shulman 2019). 230 Previous studies have shown that systemic, chronic low-grade inflammation characterized by 231 continuous elevation of inflammatory factors in the circulatory system is associated with the 232 occurrence and development of T2DM (Gardener et al. 2016; Nilholm et al. 2018). Inflammatory 233 factors such as IL-6 (Fadaei et al. 2020), TNF- $\alpha$, and CRP have been shown to predict the risk of 234 T2DM (Akour et al. 2018); however, the specific mechanism remains unclear (Naidoo et al. 235 2018). Diet may affect the development of T2DM by changing the body's inflammatory state. 236 However, prospective analysis is required to test whether this occurs in the natural history of the 237 disease.

238 Our study had considerable strengths. First, to our knowledge, this work was first to study the 239 potential of diet to promote inflammation and T2DM in Xinjiang. Second, our study was 240 conducted in the Xinjiang Uyghur Autonomous Region, located in the northwest of China, which 241 is associated with considerable ethnic and culinary heterogeneity, which could broaden the 242 distribution of DII thus making it easier to observe a true DII-T2DM relationship. This study 243 involved baseline surveys of more than 31,000 subjects in the three regions of Xinjiang and 244 provided a stable field support for the current study. Third, our participants were from multi245 ethnic populations, and therefore the association between DII and T2DM in our study was able to 246 account for potentially confounding factors related to ethnicity. Thus, we were able to show that 
247 the DII can be applied to diverse populations as described in other studies (Namazi et al. 2018;

248 Ruiz-Canela et al. 2015); and this, in turn, enables comparison with population-based findings in 249 many different regions of the world.

250 Our study also had some limitations. We used a cross-sectional design, our analysis relied on 251 observational data, and there is a chance that our findings could be explained directly by 252 causation. It was not clear whether T2DM patients were more likely to choose a pro253 inflammatory diet or whether a pro-inflammatory diet helped promote or maintain T2DM. Future 254 cohort studies are required to validate the association between T2DM and DII. As with most 255 observational studies, the FFQs in this study were self-administered; therefore, dietary intake 256 may the associated with reporting biases. In order to minimize such problems, we designed the 257 questionnaire according to local dietary conditions and used food mold during the study.

258

\section{Conclusions}

260 In summary, our study suggests that a diet with a pro-inflammatory potential is associated with 261 an increased risk of T2DM. This study included participants from the baseline study in the 262 Xinjiang multi-ethnic natural population cohort construction and health follow-up study. Our 263 results add to the understanding of the mechanisms of diet-related inflammation and T2DM. Moreover, DII may be an important tool for characterizing inflammatory diets. Finally, further longitudinal surveys are needed to assess the relationship and determine cause and effect.

266

\section{Acknowledgements}

268 The authors are indebted to all participants for their continued participation. They are also 269 grateful to all members of the Xinjiang multi-ethnic cohort study group.

270 Author Contributions: Conceptualization, W.-H.F., N.S., J.R.H. and J.-H.D.; methodology, 271 W.-H.F., N.S., J.R.H. and H.-L.P.; validation, W.-H.F., H.-L.P. and J.-H.D.; formal analysis, 272 T.L. and T.T.; investigation, W.-H.F,Z.-W.Z. and D.A.; data curation, W.-H.F, T.L. and D.A.; 273 writing (original draft preparation), W.-H.F; writing (review and editing), W.-H.F., N.S., J.R.H. 274 and J.-H.D.; supervision, N.S., J.R.H. and J.-H.D.; project administration, J.-H.D.; funding 275 acquisition, J.-H.D. All authors have read and agreed to the published version of the manuscript. 
276 Funding: This work was supported by the provincial and ministerial joint project of the State 277 Key Laboratory for the Prevention and Treatment of high morbidity in Central Asia (NO.SKL278 HIDCA-2019-), the National key research and development plan "precise medical research" key 279 special sub-project "Xinjiang multi-ethnic natural population cohort construction and health 280 follow-up study" (NO.2017YFC0907203) and Xinjiang Uygur Autonomous Region "13th Five281 Year" Key Discipline (Plateau discipline)—Public Health and Preventive Medicine. 282 283 Conflict of Interest: The authors declare that they have no conflict of interest. Dr. James R. 284 Hébert owns controlling interest in Connecting Health Innovations LLC (CHI), a company that 285 has licensed the right to his invention of the dietary inflammatory index $\left(\mathrm{DII}^{\circledR}\right)$ from the 286 University of South Carolina in order to develop computer and smart phone applications for 287 patient counseling and dietary intervention in clinical settings. Dr. Nitin Shivappa is an employee 288 of CHI. None of the authors has any conflicts of interest to declare.

289

290

291

292

293

294

295

296

297

298

299

300

301

302

303 


\section{References}

309

310

311

312

313

314

315

316

317

318

319

320

321

322

323

324

325

326

327

328

329

330

331

332

333

334

335

336

337

338

339

340

341

342

343

344

345

346

347

348

349

350

351

352

353
Akour A, Kasabri V, Bulatova N, Al Muhaissen S, Naffa R, Fahmawi H, Momani M, Zayed A, and Bustanji Y. 2018. Association of Oxytocin with Glucose Intolerance and Inflammation Biomarkers in Metabolic Syndrome Patients with and without Prediabetes. Rev Diabet Stud 14:364-371. 10.1900/RDS.2017.14.364

Alipoor E, Karimbeiki R, Shivappa N, Yaseri M, Hebert JR, and Hosseinzadeh-Attar MJ. 2019. Dietary inflammatory index and parameters of diet quality in normal weight and obese patients undergoing hemodialysis. Nutrition 61:32-37. 10.1016/j.nut.2018.09.036

Ashton MM, Dean OM, Marx W, Mohebbi M, Berk M, Malhi GS, Ng CH, Cotton SM, Dodd S, Sarris J, Hopwood M, Faye-Chauhan K, Kim Y, Dash SR, Jacka FN, Shivappa N, Hebert JR, and Turner A. 2020. Diet quality, dietary inflammatory index and body mass index as predictors of response to adjunctive $\mathrm{N}$-acetylcysteine and mitochondrial agents in adults with bipolar disorder: A sub-study of a randomised placebo-controlled trial. Aust N Z J Psychiatry 54:159-172. 10.1177/0004867419882497

Barbaresko J, Koch M, Schulze MB, and Nöthlings U. 2013. Dietary pattern analysis and biomarkers of low-grade inflammation: a systematic literature review. Nutrition Reviews 71:511-527. 10.1111/nure.12035

Cavicchia PP, Steck SE, Hurley TG, Hussey JR, Ma Y, Ockene IS, and Hebert JR. 2009. A new dietary inflammatory index predicts interval changes in serum high-sensitivity C-reactive protein. J Nutr 139:2365-2372. 10.3945/jn.109.114025

Cho NH, Shaw JE, Karuranga S, Huang Y, da Rocha Fernandes JD, Ohlrogge AW, and Malanda B. 2018. IDF Diabetes Atlas: Global estimates of diabetes prevalence for 2017 and projections for 2045. Diabetes Res Clin Pract 138:271-281. 10.1016/j.diabres.2018.02.023

Denova-Gutierrez E, Munoz-Aguirre P, Shivappa N, Hebert JR, Tolentino-Mayo L, Batis C, and Barquera S. 2018. Dietary Inflammatory Index and Type 2 Diabetes Mellitus in Adults: The Diabetes Mellitus Survey of Mexico City. Nutrients 10. 10.3390/nu10040385

Dregan A, Charlton J, Chowienczyk P, and Gulliford MC. 2014. Chronic Inflammatory Disorders and Risk of Type 2 Diabetes Mellitus, Coronary Heart Disease, and Stroke. Circulation 130:837-844. 10.1161/circulationaha.114.009990

Fadaei R, Bagheri N, Heidarian E, Nouri A, Hesari Z, Moradi N, Ahmadi A, and Ahmadi R. 2020. Serum levels of IL32 in patients with type 2 diabetes mellitus and its relationship with TNF-alpha and IL-6. Cytokine 125:154832. 10.1016/j.cyto.2019.154832

Ferreira YAM, Kravchychyn ACP, Vicente SCF, Campos R, Tock L, Oyama LM, Boldarine VT, Masquio DCL, Thivel D, Shivappa N, Hebert JR, and Damaso AR. 2019. An Interdisciplinary Weight Loss Program Improves Body Composition and Metabolic Profile in Adolescents With Obesity: Associations With the Dietary Inflammatory Index. Front Nutr 6:77. 10.3389/fnut.2019.00077

Gardener SL, Rainey-Smith SR, and Martins RN. 2016. Diet and Inflammation in Alzheimer's Disease and Related Chronic Diseases: A Review. J Alzheimers Dis 50:301-334. 10.3233/JAD-150765

Hailili G, Chen Z, Tian T, Fu W, Pei H, Mahan Y, Luo T, Alimu D, Wang L, Zhang G, Liu L, Wang D, and Dai J. 2020. Dietary patterns and their associations with metabolic syndrome and predicted 10-year risk of cardiovascular disease in northwest Chinese adults. The British journal of nutrition:1-28. 10.1017/s000711452000478x

Hebert JR, Shivappa N, Tabung FK, Steck SE, Wirth MD, and Hurley TG. 2014. On the use of the dietary inflammatory index in relation to low-grade inflammation and markers of glucose metabolism in the Cohort study on Diabetes and Atherosclerosis Maastricht (CODAM) and the Hoorn study. Am J Clin Nutr 99:1520. 10.3945/ajen.113.079095

Jacobo-Cejudo MG, Valdes-Ramos R, Guadarrama-Lopez AL, Pardo-Morales RV, Martinez-Carrillo BE, and Harbige LS. 2017. Effect of n-3 Polyunsaturated Fatty Acid Supplementation on Metabolic and Inflammatory Biomarkers in Type 2 Diabetes Mellitus Patients. Nutrients 9. 10.3390/nu9060573 
354

355

356

357

358

359

360

361

362

363

364

365

366

367

368

369

370

371

372

373

374

375

376

377

378

379

380

381

382

383

384

385

386

387

388

389

390

391

392

393

394

395

396

397

398

399

400

401

402

403

404

405
JOHN C. PICKUP D, FRCPATH. 2004. Inflammation and Activated Innate Immunity in the Pathogenesis of Type 2 Diabetes. DIABETES CARE 27:813.

Kotemori A, Sawada N, Iwasaki M, Yamaji T, Shivappa N, Hebert JR, Ishihara J, Inoue M, Tsugane S, and Group JFVS. 2020. Validating the dietary inflammatory index using inflammatory biomarkers in a Japanese population: A cross-sectional study of the JPHC-FFQ validation study. Nutrition 69:110569. 10.1016/j.nut.2019.110569

Laouali N, Mancini FR, Hajji-Louati M, El Fatouhi D, Balkau B, Boutron-Ruault MC, Bonnet F, and Fagherazzi G. 2019. Dietary inflammatory index and type 2 diabetes risk in a prospective cohort of 70,991 women followed for 20 years: the mediating role of BMI. Diabetologia 62:2222-2232. 10.1007/s00125-019-04972-0

Linden A, Mathur MB, and VanderWeele TJ. 2020. Conducting sensitivity analysis for unmeasured confounding in observational studies using E-values: The evalue package. The Stata Journal: Promoting communications on statistics and Stata 20:162-175. 10.1177/1536867x20909696

Lohman MC, Resciniti NV, Wirth MD, Shivappa N, and Hébert JR. 2019. Obesity, Dietary inflammation, and Frailty among Older Adults: Evidence from the National Health and Nutrition Examination Survey. J Nutr Gerontol Geriatr 38:18-32. 10.1080/21551197.2018.1552226

Mahlangu T, Dludla PV, Nyambuya TM, Mxinwa V, Mazibuko-Mbeje SE, Cirilli I, Marcheggiani F, Tiano L, Louw J, and Nkambule BB. 2019. A systematic review on the functional role of Th1/Th2 cytokines in type 2 diabetes and related metabolic complications. Cytokine 126:154892. 10.1016/j.cyto.2019.154892

McGeoch SC, Johnstone AM, Lobley GE, Adamson J, Hickson K, Holtrop G, Fyfe C, Clark LF, Pearson DW, Abraham P, Megson IL, and MacRury SM. 2013. A randomized crossover study to assess the effect of an oat-rich diet on glycaemic control, plasma lipids and postprandial glycaemia, inflammation and oxidative stress in Type 2 diabetes. Diabet Med 30:1314-1323. 10.1111/dme.12228

Minihane AM, Vinoy S, Russell WR, Baka A, Roche HM, Tuohy KM, Teeling JL, Blaak EE, Fenech M, Vauzour D, McArdle HJ, Kremer BH, Sterkman L, Vafeiadou K, Benedetti MM, Williams CM, and Calder PC. 2015. Low-grade inflammation, diet composition and health: current research evidence and its translation. $\mathrm{Br} \mathrm{J}$ Nutr 114:999-1012. 10.1017/S0007114515002093

Moazzen S, van der Sloot KWJ, Vonk RJ, de Bock GH, and Alizadeh BZ. 2020. Diet Quality and Upper Gastrointestinal Cancers Risk: A Meta-Analysis and Critical Assessment of Evidence Quality. Nutrients 12. 10.3390/nu12061863

Mtintsilana A, Micklesfield LK, Chorell E, Olsson T, Shivappa N, Hebert JR, Kengne AP, and Goedecke JH. 2019. Adiposity Mediates the Association between the Dietary Inflammatory Index and Markers of Type 2 Diabetes Risk in Middle-Aged Black South African Women. Nutrients 11. 10.3390/nu11061246

Naidoo V, Naidoo M, and Ghai M. 2018. Cell- and tissue-specific epigenetic changes associated with chronic inflammation in insulin resistance and type 2 diabetes mellitus. Scand J Immunol 88:e12723. 10.1111/sji.12723

Namazi N, Larijani B, and Azadbakht L. 2018. Dietary Inflammatory Index and its Association with the Risk of Cardiovascular Diseases, Metabolic Syndrome, and Mortality: A Systematic Review and Meta-Analysis. Horm Metab Res 50:345-358. 10.1055/a-0596-8204

Nilholm C, Roth B, Hoglund P, Blennow K, Englund E, Hansson O, Zetterberg H, and Ohlsson B. 2018. Dietary intervention with an Okinawan-based Nordic diet in type 2 diabetes renders decreased interleukin-18 concentrations and increased neurofilament light concentrations in plasma. Nutr Res 60:13-25. 10.1016/j.nutres.2018.08.002

Oliveira TMS, Bressan J, Pimenta AM, Martinez-Gonzalez MA, Shivappa N, Hebert JR, and Hermsdorff HHM. 2019. Dietary inflammatory index and prevalence of overweight and obesity in Brazilian graduates from the Cohort of Universities of Minas Gerais (CUME project). Nutrition 71:110635. 10.1016/j.nut.2019.110635

Padin AC, Hebert JR, Woody A, Wilson SJ, Shivappa N, Belury MA, Malarkey WB, Sheridan JF, and Kiecolt-Glaser JK. 2019. A proinflammatory diet is associated with inflammatory gene expression among healthy, nonobese adults: Can social ties protect against the risks? Brain Behav Immun 82:36-44. 10.1016/j.bbi.2019.07.031

Phillips CM, Chen LW, Heude B, Bernard JY, Harvey NC, Duijts L, Mensink-Bout SM, Polanska K, Mancano G, Suderman M, Shivappa N, and Hebert JR. 2019. Dietary Inflammatory Index and Non-Communicable Disease Risk: A Narrative Review. Nutrients 11:1873-1905. 10.3390/nu11081873

Pradhan A D, Manson J E, Rifai N, and JE B. 2001. C-reactive protein, interleukin 6, and risk of developing type 2 diabetes mellitus. JAMA 286:327-334.

Peer) reviewing PDF | (2020:10:53822:2:1:NEW 2 Mar 2021) 
406 407 408 409 410 411

412

413

414

415

416

417

418

419

420

421

422

423

424

425

426

427

428

429
Roden M, and Shulman GI. 2019. The integrative biology of type 2 diabetes. Nature 576:51-60. 10.1038/s41586-0191797-8

Ruiz-Canela M, Zazpe I, Shivappa N, Hebert JR, Sanchez-Tainta A, Corella D, Salas-Salvado J, Fito M, LamuelaRaventos RM, Rekondo J, Fernandez-Crehuet J, Fiol M, Santos-Lozano JM, Serra-Majem L, Pinto X, Martinez JA, Ros E, Estruch R, and Martinez-Gonzalez MA. 2015. Dietary inflammatory index and anthropometric measures of obesity in a population sample at high cardiovascular risk from the PREDIMED (PREvencion con DIeta MEDiterranea) trial. Br J Nutr 113:984-995. 10.1017/S0007114514004401

Shivappa N, Steck SE, Hurley TG, Hussey JR, and Hebert JR. 2014. Designing and developing a literature-derived, population-based dietary inflammatory index. Public Health Nutr 17:1689-1696. 10.1017/S1368980013002115

Shivappa N, Wirth MD, Murphy EA, Hurley TG, and Hebert JR. 2019. Association between the Dietary Inflammatory Index (DII) and urinary enterolignans and C-reactive protein from the National Health and Nutrition Examination Survey-2003-2008. Eur J Nutr 58:797-805. 10.1007/s00394-018-1690-5

Tabung FK, Steck SE, Zhang J, Ma Y, Liese AD, Agalliu I, Hingle M, Hou L, Hurley TG, Jiao L, Martin LW, Millen AE, Park HL, Rosal MC, Shikany JM, Shivappa N, Ockene JK, and Hebert JR. 2015. Construct validation of the dietary inflammatory index among postmenopausal women. Ann Epidemiol 25:398-405. 10.1016/j.annepidem.2015.03.009

Wang H P, Yao J J, Yin X, and L GX. 2020. Organisational and individual characteristics associated with glycaemic control among patients with type 2 diabetes cross-sectional study in China. BMJ Open 10:e036331. 10.1136/

Zhong X, Guo L, Zhang L, Li Y, He R, and Cheng G. 2017. Inflammatory potential of diet and risk of cardiovascular disease or mortality: A meta-analysis. Scientific Reports 7:6367-6373. 10.1038/s41598-017-06455-x

Zuo H, Shi Z, and Hussain A. 2014. Prevalence, trends and risk factors for the diabetes epidemic in China: A systematic review and meta-analysis. Diabetes Research and Clinical Practice 104:63-72. 10.1016/j.diabres.2014.01.002 


\section{Table 1 (on next page)}

Characteristics of the study population 


\section{Table 1:}

2 Characteristics of the study population

\begin{tabular}{ccccc}
\hline & $\begin{array}{c}\text { Overall } \\
\text { Study }\end{array}$ & $\begin{array}{c}\text { Non-T2DM } \\
\text { Subjects }\end{array}$ & $\begin{array}{c}\text { T2DM } \\
\text { Subjects }\end{array}$ & \\
\cline { 2 - 4 }$(\mathrm{n}=5105)$ & $(\mathrm{n}=4686)$ & $(\mathrm{n}=419)$ & \\
\hline Sex, $n(\%)$ & & & & \\
Male & $2998(58.7)$ & $2769(59.1)$ & $229(54.7)$ & 0.08 \\
Female & $2107(41.3)$ & $1917(40.9)$ & $190(45.4)$ & \\
\hline Age groups, $n(\%)$ & & & & \\
$<45$ & $1662(32.6)$ & $1605(34.3)$ & $57(13.6)$ & \\
$45-60$ & $2323(45.5)$ & $2132(45.5)$ & $191(45.6)$ & \\
$>60$ & $1120(22.0)$ & $949(20.3)$ & $171(40.8)$ & \\
BMI $<24 \mathrm{~kg} / \mathrm{m}^{2}$ & $1584(31.0)$ & $1462(31.2)$ & $122(29.1)$ & \\
BMI $24-28 \mathrm{~kg} / \mathrm{m}^{2}$ & $1837(36.0)$ & $1678(35.8)$ & $164(37.9)$ & \\
BMI $>28 \mathrm{~kg} / \mathrm{m}^{2}$ & $1684(33.0)$ & $1546(33.0)$ & $138(32.9)$ & \\
\hline
\end{tabular}

Ethnicity, $n(\%)$

$\begin{array}{ccccc}\text { Han } & 665(13.0) & 590(12.6) & 75(17.9) & <0.001 \\ \text { Kazak } & 919(18.0) & 879(18.8) & 40(9.6) & \\ \text { Hui } & 1302(25.5) & 1153(24.6) & 149(35.6) & \\ \text { Uyghur } & 746(14.6) & 661(14.1) & 85(20.3) & \\ \text { Others } & 1473(28.9) & 1403(29.9) & 70(16.7) & \\ \text { Educational level, } n(\%) & & & & \\ \text { Low } & 3695(73.1) & 3378(72.8) & 317(73.1) & 0.34 \\ \text { Medium } & 1337(26.4) & 1237(26.7) & 100(23.9) & \\ \text { High } & 25(0.5) & 24(0.5) & 1(0.2) & \\ \text { Smoking status, } n(\%) & & & & \\ \text { Never } & 3980(78.0) & 3643(77.7) & 337(80.4) & 11(2.6) \\ \text { Once in a while } & 106(2.1) & 95(2.0) & 71(17.0) & \\ \text { Usually } & 1019(20.0) & 948(20.0) & & \\ \text { Alcohol status, } n(\%) & & & 367(87.5) & 0.05 \\ \text { Never } & 4300(84.2) & 3933(83.9) & 61(9.8) & \\ \text { Once in a while } & 686(13.4) & 645(13.8) & 11(2.6) & \\ \text { Usually } & 119(2.3) & 108(2.3) & \end{array}$

Physical activity level, $n(\%)$

Low $\quad 2862(56.1) \quad 2571(54.9) \quad 291(69.5) \quad<0.001$




\begin{tabular}{ccccc} 
Medium & $2106(41.3)$ & $1983(42.3)$ & $123(29.4)$ & \\
High & $137(2.7)$ & $132(2.8)$ & $5(1.2)$ & \\
\hline Glucose(mmol/L) & $5.12(0.02)$ & $4.85(0.01)$ & $8.10(0.2)$ & $<0.001$ \\
\hline DII & $0.81(0.1)$ & $0.76(0.1)$ & $1.44(0.2)$ & $<0.001$ \\
\hline
\end{tabular}

3 
Table 2 (on next page)

Baseline characteristics of study population by Dietary Inflammatory Index(DII) categorization 
1 Table 2:

2 Baseline characteristics of study population by Dietary Inflammatory Index(DII)

3 categorization

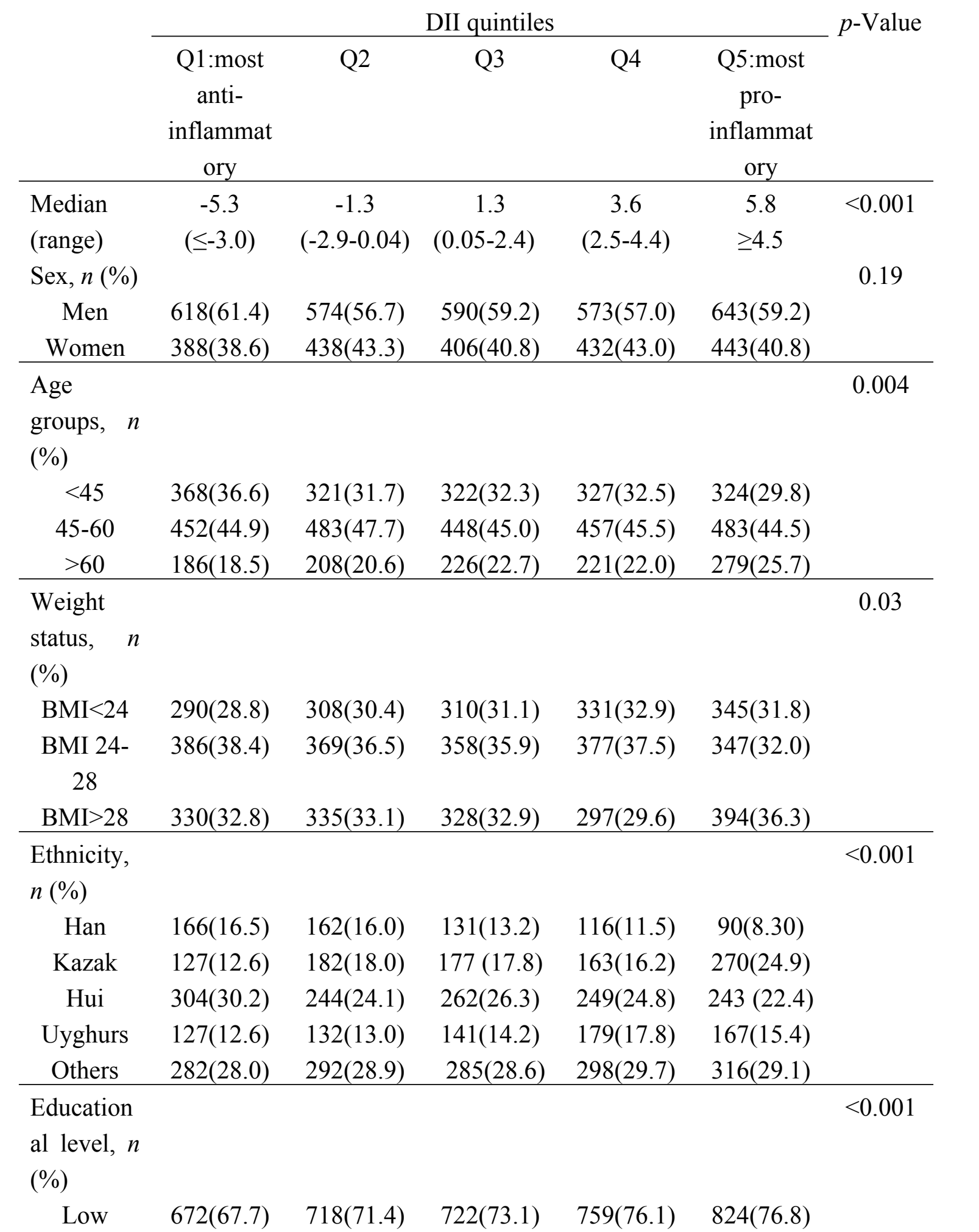




\begin{tabular}{|c|c|c|c|c|c|c|}
\hline $\begin{array}{c}\text { Medium } \\
\text { High }\end{array}$ & $\begin{array}{c}316(31.8) \\
5(0.5)\end{array}$ & $\begin{array}{c}283(28.1) \\
5(0.5)\end{array}$ & $\begin{array}{c}259(26.2) \\
7(0.7)\end{array}$ & $\begin{array}{c}235(23.6) \\
3(0.3)\end{array}$ & $\begin{array}{c}244(22.7) \\
5(0.5)\end{array}$ & \\
\hline $\begin{array}{l}\text { Smoking } \\
\text { status, } n \\
(\%)\end{array}$ & & & & & & 0.47 \\
\hline Never & $722(76.7)$ & $801(79.2)$ & $779(78.2)$ & $795(79.1)$ & 833 (76.7) & \\
\hline $\begin{array}{c}\text { Once in a } \\
\text { while }\end{array}$ & $27(2.7)$ & $18(1.8)$ & $22(2.2)$ & $22(2.2)$ & $17(1.6)$ & \\
\hline Usually & $207(20.6)$ & $193(19.1)$ & 195(19.6) & $188(18.7)$ & $236(21.7)$ & \\
\hline $\begin{array}{l}\text { Alcohol } \\
\text { status, } n \\
(\%)\end{array}$ & & & & & & 0.20 \\
\hline Never & $826(82.1)$ & $849(83.9)$ & $842(84.5)$ & $864(86.0)$ & 919 (84.7) & \\
\hline $\begin{array}{c}\text { Once in a } \\
\text { while }\end{array}$ & $149(14.8)$ & $136(13.4)$ & $136(13.7)$ & $122(12.1)$ & $143(13.2)$ & \\
\hline Usually & $31(3.1)$ & $27(2.7)$ & $18(1.8)$ & 19(1.9) & $24(2.2)$ & \\
\hline $\begin{array}{l}\text { Physical } \\
\text { activity } \\
\text { level, } \\
n(\%)\end{array}$ & & & & & & 0.002 \\
\hline Low & $538(53.5)$ & $566(55.9)$ & $534(53.6)$ & $573(57.0)$ & $651(59.9)$ & \\
\hline Medium & $435(43.2)$ & $421(41.6)$ & $447(44.9)$ & $396(39.4)$ & $407(37.5)$ & \\
\hline High & $33(3.3)$ & $25(2.5)$ & $15(1.5)$ & $36(3.6)$ & $28(2.6)$ & \\
\hline $\begin{array}{l}\text { Glucose } \\
(\mathrm{mmol} / \mathrm{L}), \\
\text { mean }(\mathrm{SD})\end{array}$ & $4.98(1.0)$ & $5.03(1.1)$ & $4.99(1.0)$ & $5.00(1.0)$ & $5.57(3.0)$ & $<0.001$ \\
\hline
\end{tabular}




\section{Table 3(on next page)}

Results of multivariate logistic regression models examining the relation between dietary inflammatory index and T2DM 
1 Table 3:

2 Results of multivariate logistic regression models examining the relation between dietary

3 inflammatory index and T2DM

\begin{tabular}{|c|c|c|c|c|c|c|c|}
\hline & \multirow{2}{*}{$\begin{array}{c}\text { No. } \\
\text { T2DM } \\
(\%)\end{array}$} & \multicolumn{2}{|c|}{ Unadjusted } & \multicolumn{2}{|c|}{$\begin{array}{l}\text { Sex- and Ethnicity- } \\
\text { Adjusted }\end{array}$} & \multicolumn{2}{|c|}{ Fully Adjusted } \\
\hline & & $\mathrm{OR}(95 \% \mathrm{CI})$ & $p$ & $\mathrm{OR}(95 \% \mathrm{CI})$ & $p$ & $\mathrm{OR}(95 \% \mathrm{CI})$ & $p$ \\
\hline $\begin{array}{l}\text { DII as continuous } \\
\text { variable per }\end{array}$ & 419 & $1.05(1.02,1.08)$ & 0.001 & $1.06(1.03,1.08)$ & $<0.001$ & $1.05(1.02,1.08)$ & $<0.001$ \\
\hline 1-piont increase & & & & & & & \\
\hline $\begin{array}{l}\text { DII as categorical } \\
\text { variable }\end{array}$ & & & & & & & \\
\hline Q1 & 60 & 1(reference) & & 1(reference) & & 1(reference) & \\
\hline Q2 & 63 & $1.05(0.73,1.51)$ & 0.81 & $1.12(0.77,1.62)$ & 0.55 & $1.12(0.77,1.62)$ & 0.55 \\
\hline Q3 & 65 & $1.10(0.77,1.58)$ & 0.60 & $1.17(0.82,1.69)$ & 0.39 & $1.14(0.79,1.65)$ & 0.48 \\
\hline Q4 & 66 & $1.11(0.77,1.59)$ & 0.58 & $1.18(0.82,1.70)$ & 0.37 & $1.15(0.80,1.66)$ & 0.46 \\
\hline Q5 & 165 & $2.83(2.07,3.85)$ & $<0.001$ & $3.37(2.46,4.63)$ & $<0.001$ & $3.27(2.38,4.50)$ & $<0.001$ \\
\hline
\end{tabular}


Figure 1

Flow diagram of subjects included in the study 


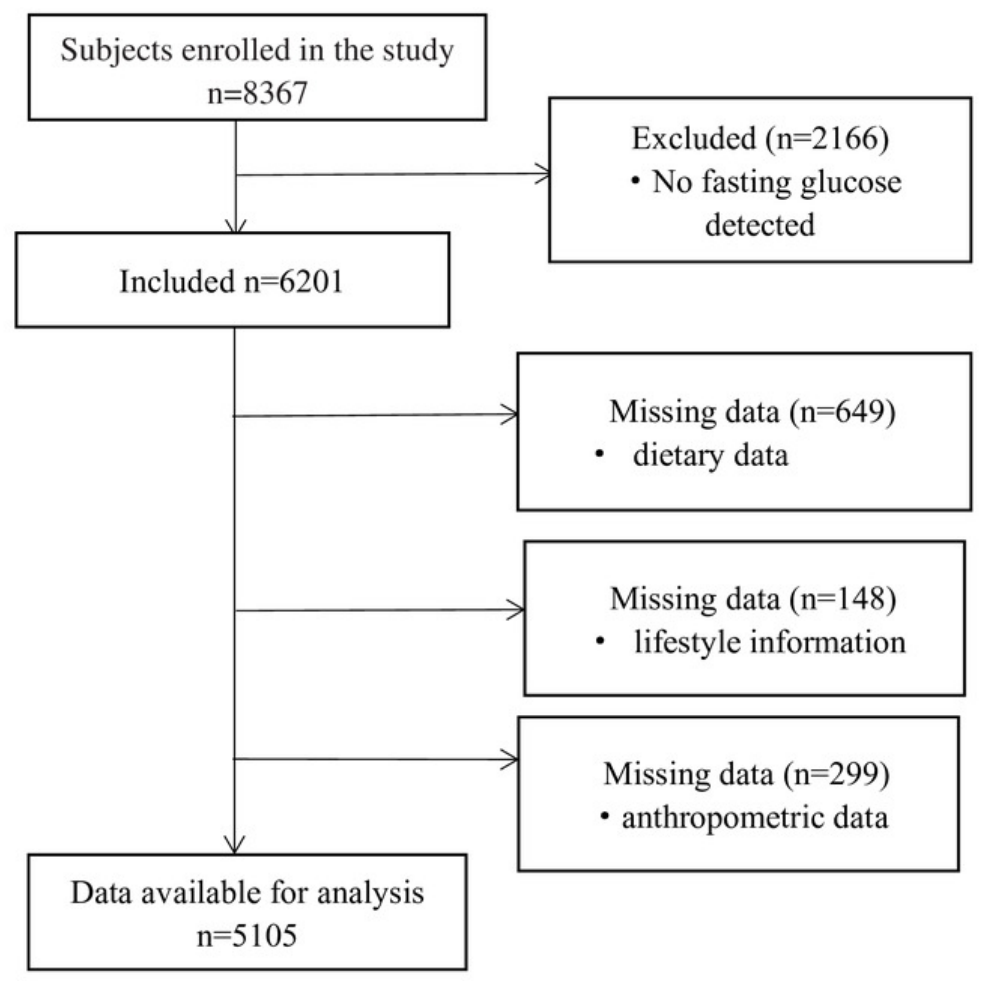


Figure 2

Odds ratio $(95 \% \mathrm{Cl})$ of Dietary Inflammatory Index (DII) and T2DM 


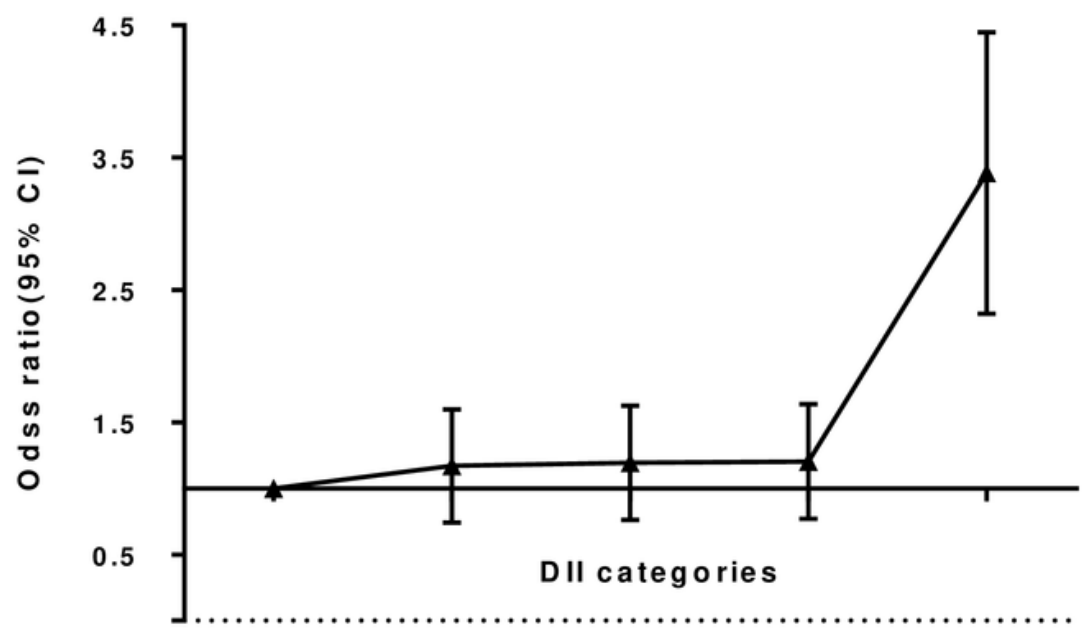

$\begin{array}{llllll}\text { DII } & \text { Q1 } & \text { Q2 } & \text { Q3 } & \text { Q4 } & \text { Q5 }\end{array}$

No. of patients 60

63

65

66

165 
Figure 3

Stratified analysis of the association between DII and T2DM after adjusting for potential confounding factors (Quartile 5 vs. Quartile 1). 


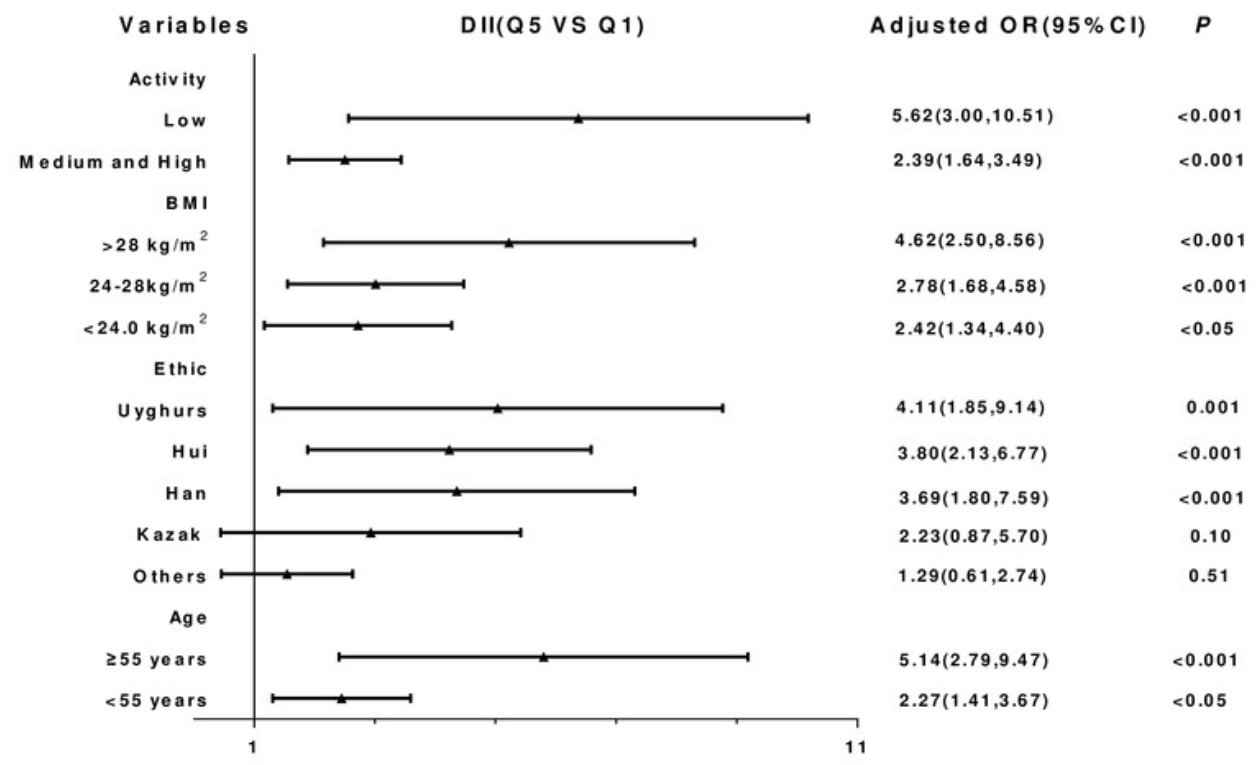

